\title{
The Solution to Graviton as Goldstone Boson
}

\begin{abstract}
Aayush Verma
The study of Graviton as Goldstone bosons appeared in the 1960s, after Bjorken interacting idea of Electrodynamics. However, no recent advancement has been done in the field, because of very constraints as well as low-attractiveness of the theory. We do the non-metric tensor (covariant derivative of the metric tensor) case of Gravitation and eventually get $\mathrm{SO}(1,3)$ broken in the vacuum state of quantized field theory, then find the Graviton as Goldstone Boson. We, in final, see that Gravitons can have appearances in many modified (and extended) theories of Gravitation.
\end{abstract}

Keywords: Gravitation; Graviton; BEH Mechanism

\section{INTRODUCTION}

Graviton as Goldstone Boson is an idea established by Peter Phillips [1], later modified using fermionic fields by Hans Ohanian [2]. Both used the Bjorken [3] dynamical style of those massless photons, which perhaps is a wonderful lying in early Quantum Field Theory.

For a Goldstone 4, one have a Lagrangian showing some system and obeying a continuous symmetry, but that symmetry doesn't leave ground state invariant, then one gets a massless particle as boson from that system.

We know that Photon is a Goldstone boson in Quantum Electrodynamics (QED), traveling with the speed of light. Graviton, too, can be treated as a Goldstone boson, this can be done if one shows that the ground state breaks some kind of continuous symmetry. Lorentz Symmetry is that symmetry, by some authors. The procedure imitates the broken symmetry that is seen in QED.

In fact, one gets the Graviton from the fermionic field [2], by taking tensor-tensor coupling as a currentcurrent coupling in QED. These tensors have vacuum expectation value (v.e.v) $\neq 0$, which instantly breaks the Lorentz Symmetry and giving Gravitons as poles in S-Matrix. However, a lot of requirements are needed to make this model workable, like linearity in the system, hence not producing any complex non-linear renormalizable high-order interactions. However, one can simply get linear high-order Feynman interactions with four-fermions interaction, but that is not much convenient and advisable, because of the cut-off, however one can have the S-Matrix out of such complex systems of bound states using the imitation of partition functional and Gell-Mann and Low theorem [5] of green functions, but don't know-how.

Here, in the note, we try to put out a few results based on a non-metric tensor Lagrangian and getting Gravitons as Goldstone Boson. This may be unique, as only non-covariant tensors are preferred in the gauge choice. But here, we try to implement the non-metric $\mathrm{f}(\mathrm{Q})$ metric (only to a particular advancement) in a very generalized yet not pure way. Our main task, which is though very simple to show that symmetry breaks in a non-metric system, and then we know most of the extended versions of Gravitation can imitate the result, and get Gravitons appearing as Goldstone Boson.

\section{THE OHANION AND PHILLIPS METHOD}

First we ought to discuss Phillips [1] and Ohanian [2] perspective and methods, through which they reached the linear results, in the light of Electrodynamics.

Peter Phillips, outlined a theoretical model in which Lorentz Symmetry is spontaneously violated, giving rise to some vector fields $\lambda^{\mu}$. Starting with a metric in quantize Lagrangian, where he write a propagator ${ }^{1}$

$$
E_{\mu \nu}^{\sigma \rho}(p)=-\frac{i \gamma}{2}\left(g_{\mu}^{\sigma} g_{\nu}^{\rho}+g_{\nu}^{\sigma} g_{\mu}^{\rho}\right)+\gamma \Xi_{\mu \nu}^{\alpha \beta}(q) \Xi_{\alpha \beta}^{\sigma \rho}(q)
$$

where $E_{\mu \nu}^{\sigma \rho}(p)$ is the graviton propagator, $\gamma$ is a coupling constant, don't have linear dependence on Gravitational constant, $\Xi_{\mu \nu}^{\alpha \beta}(q)$ is the graviton self-energy. However, his calculations to propagator and graviton (Gravitational tensors), are good for a self-basis field and is basically dependent on the Renormalization, with cutoffs. We don't need to continue our discussions on this now, because the model is only self-consistent in the matter of $\Lambda$.

Hans Ohanian, took a further step, introducing graviton as goldstone boson from a self-interacting fermionic field. Now, this model is splendid in the matter of physical solutions to a lot of problems, Bethe-Salpeter equation, and successfully quantizes the Gravitational theory, at least in the linear order form.

In [2], the field theory calculations have been done in a good way, finally producing v.e.v, with non-trivial

\footnotetext{
1 However, this is very imitating to original momentum-space version of Bjorken propagator. But, the tensors are introduced because a Lagrangian can't start without a tensor field in gravitational model unlike in electrodynamics.
} 
solutions, hence giving gravitons as Goldstone bosons, in potential $\mathrm{V}$.

A tensor $t_{\mu \nu}$ is introduced as formal energy-momentum tensor, in order to break the symmetry

$$
\left\langle 0^{\prime}\left|t_{\mu \nu}\right| 0^{\prime}\right\rangle=0 \neq C g_{\mu \nu}
$$

where

$$
t_{\mu \nu}=T_{\mu \nu}-\frac{1}{4} g_{\mu \nu}\left\langle 0^{\prime}|T| 0^{\prime}\right\rangle
$$

and $T_{\mu \nu}$ is introduced here as a coupling between fields, as it is a fermionic theory

$$
T_{\mu \nu}=\frac{1}{2} i\left(\bar{\psi} \gamma_{\mu} \partial_{v}-\partial_{v} \gamma \mu \psi\right.
$$

and $\left|0^{\prime}\right\rangle$ is a vacuum, that is why the Eq. (2), is introduced. The Lagrangian for a single spinor field from where the model starts with is

$$
\mathcal{L}=\frac{1}{2} \bar{\psi}\left(i \gamma_{\mu} \partial_{\mu}-m\right) \psi+\frac{1}{2} K\left(t^{\mu \nu} t_{\mu \nu}-a t_{\mu}^{\mu} t_{\nu}^{\mu}\right)+H . D
$$

where $\mathrm{K}$ is derived using Hartee-Self consistency condition and $\mathrm{a}$ is some other constant. And then the model proceeds with lots of S-Matrix, getting the non-perturbation in first and then getting the gravitons as propagators, internal lines from the fermionic interactions. However, in spite of Bludman 6, explanation to these bosons as just gauge excitation or not even real physical particles, we can appreciate the model.

Now we will be in need of some concepts from Ohanian method and [7]. But we will follow a different perspective, but however same practise.

\section{GRAVITATION, TENSOR AND GRAVITONS}

Now moving to the main work. First, we need a quantized Lagrangian, which we will borrow from [7], because of insightful choice. However we will replace the metric with a non-metric system.

$$
\begin{array}{r}
\mathcal{L}=A\left(\Lambda^{2} \sqrt{-\nabla_{\alpha} g_{\mu \nu}} f(Q)-\Lambda^{4} V(h)+\text { H.O.D }+\right. \\
\left.\mathcal{L}_{\text {matter }}+\mathcal{O}\left(A^{\circ}\right)\right)
\end{array}
$$

A is some constant (normalizing), $\nabla_{\alpha} g_{\mu \nu}$ is the covariant derivative, as we are working in non-metric, nonRiemannian space-time, $\mathrm{V}(\mathrm{h})$ is our potential, H.O.D is higher order derivatives and then $\mathcal{L}_{\text {matter }}$ is some perturbed Lagrangian, and $\mathrm{f}(\mathrm{Q})$ [8, 9, 10, is symmetric modified gravity tensor and $\mathrm{Q}$ depicts the non-metricity. However $\mathrm{f}(\mathrm{Q})$, should be restricted in the sense as a modified telleparallel gravity, which is not discuss here. The reasons for taking these nonmetricity tensors, is that this will provide us non-linear solutions (but also very diverging), in contrast to previous works. And in non-Riemannian space-time, as for say, Weitzenböck space [1], we have much to process for these kinds of Lagrangian, which we will not do here.

We will say,

$$
Q_{\alpha \mu \nu}=\nabla_{\alpha} g_{\mu \nu}
$$

and $g_{\mu \nu}=n_{\mu \nu}+h_{\mu \nu}$. We now introduce a tensor, which is capable of breaking the symmetry at ground state, $t_{\mu \nu}$, which we say is equal to $G_{\mu \nu} \nabla_{\alpha} h_{\mu \nu}, G_{\mu \nu}$ is some other tensor, which is proportional to $g_{\mu \nu}$. And $t_{\mu \nu}$ is defined in such way as Eq (2)

$$
\left\langle 0^{\prime}\left|t_{\mu \nu}\right| 0^{\prime}\right\rangle=\left\langle t_{\mu \nu}\right\rangle \neq C Q_{\alpha \mu \nu}
$$

that defines the vacuum state breaking the symmetry. However, we will be seeing other way to check this breaking mechanism, with a $\mathrm{SO}(1,3)$ generator $J^{\mu \nu}$. What now should concern us is $\left\langle\nabla_{\alpha} h_{\mu \nu}\right\rangle$ (the vacuum state), so we now want our $\nabla_{\alpha} h_{\mu \nu}$ to be broken as Eq (2). Doing it in nonmetricity shouldn't change anything, but the quantized QFT particle. As Eq 8)

$$
\left\langle G_{\mu \nu} \nabla_{\alpha} h_{\mu \nu}\right\rangle \neq C Q_{\mu \nu}
$$

in other words (in rigid sense, eliminating $G_{\mu \nu}$ )

$$
\left\langle\nabla_{\alpha} h_{\mu \nu}\right\rangle J^{\mu \nu} \neq\left\langle\nabla_{\alpha} h_{\mu \nu}\right\rangle
$$

in order to proof that, we could use the Eq (8), then we just need to find the $G_{\mu \nu}$, which in fact can be set to very less (or 1, because of the proportionality constant nature here, but we don't need to do it). Then it would be something,

$$
\left\langle G_{\mu \nu} \nabla_{\alpha} h_{\mu \nu}\right\rangle
$$

which we hope to write in diogonalized form

$$
\left(\begin{array}{cccc}
\left\langle G_{11} \nabla_{\alpha} h_{11}\right\rangle & 0 & 0 & 0 \\
0 & \left\langle G_{22} \nabla_{\alpha} h_{22}\right\rangle & 0 & 0 \\
0 & 0 & \left\langle G_{33} \nabla_{\alpha} h_{33}\right\rangle & 0 \\
0 & 0 & 0 & \left\langle G_{44} \nabla_{\alpha} h_{44}\right\rangle
\end{array}\right)
$$

Now we would be wanting to calculate

$$
\begin{aligned}
{\left[G_{\mu \nu}, \nabla_{\alpha} h_{\mu \nu}\right] } & =G_{\mu \nu} \nabla_{\alpha} h_{\mu \nu}-\nabla_{\alpha} h_{\mu \nu} G_{\mu \nu} \\
& =N g_{\mu \nu} \nabla_{\alpha} h_{\mu \nu}-\nabla_{\alpha} h_{\mu \nu} g_{\mu \nu} N
\end{aligned}
$$

where $\mathrm{N}$ is a constant (better be differential) linking $G_{\mu \nu}$ and $g_{\mu \nu}$. Expanding further and simplifying more with $g_{\mu \nu}=n_{\mu \nu}+h_{\mu \nu}$

$$
N n_{\mu \nu} \nabla_{\alpha} h_{\mu \nu}+h_{\mu \nu} \nabla_{\alpha} h_{\mu \nu}-\nabla_{\alpha} h_{\mu \nu} n_{\mu \nu}-\nabla_{\alpha} h_{\mu \nu} h_{\mu \nu} N
$$

now we will make more simplifications in order to make life more easier,

$$
\begin{aligned}
n_{\mu \nu} \nabla_{\alpha} h_{\mu \nu} & =\gamma_{\mu \nu} \\
h_{\mu \nu} \nabla_{\alpha} h_{\mu \nu} & =\lambda_{\mu \nu} \\
\nabla_{\alpha} h_{\mu \nu} n_{\mu \nu} & =\gamma_{\mu \nu}^{\prime} \\
\nabla_{\alpha} h_{\mu \nu} h_{\mu \nu} & =\lambda_{\mu \nu}^{\prime}
\end{aligned}
$$


then because the commutation relation doesn't commute, and with new notation

$$
N \gamma_{\mu \nu}+\lambda_{\mu \nu}-\gamma_{\mu \nu}^{\prime}-\lambda_{\mu \nu}^{\prime} N=\beta
$$

and $\mathrm{J}$ is commutator, because we have simplified the way in peaceful and simple manner, we say

$$
N \gamma_{\mu \nu}+\lambda_{\mu \nu}=\gamma_{\mu \nu}^{\prime}+\lambda_{\mu \nu}^{\prime} N+\beta
$$

the left hand side is same as $G_{\mu \nu} \nabla_{\alpha} h_{\mu \nu}$, it is evident, then Eq 11] can be written as

$$
\left\langle\gamma_{\mu \nu}^{\prime}+\lambda^{\prime} N_{\mu \nu}+\beta\right\rangle
$$

and because of the non-vanishing value seen (in the form of beta) there and $J^{\mu \nu}$ is a generator of $\mathrm{SO}(1,3)$,

$$
\left\langle\gamma_{\mu \nu}^{\prime}+\lambda^{\prime} N_{\mu \nu}+\beta\right\rangle J^{\mu \nu} \neq\left\langle\gamma_{\mu \nu}^{\prime}+\lambda^{\prime} N_{\mu \nu}+\beta\right\rangle
$$

and then, Eq 10 is satisfied. And the vacuum state, do get symmetry broken. Hence by simply interpreting the Nambu-Goldstone theorem, we can say that the Lagrangian if properly quantized, may give us graviton which acts as Goldstone boson in non-metric space, that we have just computed.

It must be carefully noticed that the thing we computed is not the precise ground state, rather a generalization with connection to the pure Minkowski Metric. However, one can derive the simple, non-metric covariant metric solution as well, it will be needed then to calculate the pure ground state. By not "pure", I must defend that I am referring to a generalized solution rather than an exact solution to the non-metric choice problem. Theoretically, one must appreciate the things outside the normal Riemann Surface, but with interpretations, these models can be regarded as just models, as long as we don't find the non-linear solutions to be encoded precisely in our calculations of particle physics more often.

We have done our calculations. Now calculating an S-Matrix for these gravitons in fermionic or bosonic diagrams would be more than complicated because of non-linear terms. So, we don't need to do it. However, we can seek a simple propagator of this kind.

If one gets particular questions regarding the validity of these Goldstone bosons, I must point out that the calculations based on covariant mathematics, don't get hindrance with Goldstone boson, if properly done. Indeed, as pointed by G. S. Guralnik et.al. [12].

\section{CONCLUSION}

Now, in conclusion, we did a calculation based on a non-metric space (but not the Reinmanian one) and deliberating a little we got our vacuum state to be infected by the loss of invariance, which resulted in Gravitons as Goldstone bosons. What this implies that these Gravitons, as in Ohanian's or Phillip's models if appeared to be right, then they must be in some other generalization of space, that may include extensions of normal Ricci Tensors.

[1] P. R. Phillips, Physical Review 146, 966 (1966).

[2] H. C. Ohanian, Physical Review 184, 1305 (1969).

[3] J. D. Bjorken, Ann. Phys. 24, 174 (1963).

[4] J. Goldstone, A. Salam, and S. Weinberg, Physical Review 127, 965 (1962).

[5] M. Gell-Mann and F. Low, Physical Review 84, 350 (1951).

[6] S. A. Bludman, (W. A. Benjamin, Inc., New York,) (1966).

[7] P. Kraus and E. Tomboulis, Physical Review D 66, 045015 (2002).

[8] A. Conroy and T. Koivisto, The European Physical Journal C 78, 923 (2018).

[9] H. A. Buchdahl, Monthly Notices of the Royal Astronomical Society 150, 1 (1970).

[10] Y. Xu, G. Li, T. Harko, and S.-D. Liang, The European Physical Journal C 79, 708 (2019).

[11] Z. Haghani, T. Harko, H. R. Sepangi, and S. Shahidi, Journal of Cosmology and Astroparticle Physics 2012 (10), 061.

[12] G. S. Guralnik, C. R. Hagen, and T. W. Kibble, Physical Review Letters 13, 585 (1964). 